\title{
Location results: an under used tool in higher order boundary value problems
}

\author{
Feliz Minhós \\ University of Évora, Department of Mathematic. Research Centre in Mathematic and Applications \\ (CIMA-UE). Rua Romão Ramalho, 59. 7000-671 Évora. PORTUGAL.
}

\begin{abstract}
The method of lower and upper solutions provides, as well as results of existence, other important properties such as location of solution, extremal solutions,..., which have been under used and, moreover, its potential has not been optimized, either in theory either in applications. This work will present some cases to emphasize both items: two fourth order problems with functional boundary conditions (including an application to a continuous model for the deformation of the human spine under the action of some forces) and a third order periodic problem where unbounded nonlinearities are allowed, provided that an one-sided Nagumo-type condition is verified.
\end{abstract}

Keywords: Nonordered lower and upper solutions, location result, third order periodic problems, functional boundary conditions

PACS: $02.30 . \mathrm{Hq}, 02.30 . \mathrm{Sa}, 02.30 . \mathrm{Tb}, 02.70 . \mathrm{Pt}$

\section{INTRODUCTION}

Lower and upper solutions method is often used to prove the existence and/or multiplicity of solution for several types of boundary value problems in different areas, theory and applications of Mathematic: in continuous and discrete cases, ordinary and partial differential equations, problems in time scales, differential inclusions,...

However, for higher order boundary value problems, this method provides an additional tool: location data. Indeed, this information, which has not been optimized, depends of the type of lower and upper functions to be considered. The assumption of "sharp" lower and upper solutions can give more information about the type and the behavior of solutions (sign, variation, convexity,...). Let us mentioned that the iterative technique can even provide the sharpest lower and upper functions, given by the extremal solutions (maximal and minimal solutions (see, for instance, [2] and the references therein).

This paper is organized in the following way: Section 2 contains a fourth order problem composed by a nonlinear fully fourth order equation and functional boundary conditions, which include several types of situations such as separated and multi-point ones or with maximum and minimum conditions, widespread, some how, $[1,2,3,4$, 11]. The information provided by the location part is emphasized by an example to a generalized multi-point problem and an application to a continuous model, to study the impact caused by some forces in the human spine deformation (aircraft ejections, vehicle crash situations, heavy bags on children's back,...).

In Section 3 it is presented a third order periodic problem in presence of non ordered lower and upper solutions, suggested in [6]. However, the current equation assumes an

CP1124, Mathematical Models in Engineering, Biology, and Medicine, Proceedings of the

International Conference on Boundary Value Problems, edited by A. Cabada, E. Liz, and J. J. Nieto

(C) 2009 American Institute of Physics 978-0-7354-0660-5/09/\$25.00 
unilateral Nagumo-type condition ([5]) which allows to include unbounded nonlinearities. Therefore it improves the existent results in the literature, such as $[8,9,10]$. Moreover, an example will show how the location part, inherent to theorems resulting from the application of this method, can be useful to know, at least, a value of the solution and to prove the existence of infinite non-constant solutions.

\section{LOCATION RESULTS: AN UNDER USED TOOL}

This section contains an existence and location theorem applied to a generalized multipoint problem and a continuous model of the spine, where the localization of solution and its derivatives is useful to conclude some features of the solution.

\subsection{Fourth order functional problem}

To illustrate these existence and location results, it is referred the fourth order problem composed by

$$
u^{(i v)}(x)=f\left(x, u(x), u^{\prime}(x), u^{\prime \prime}(x), u^{\prime \prime \prime}(x)\right)
$$

with $x \in I:=[0,1], f: I \times \mathbb{R}^{4} \rightarrow \mathbb{R}$ a continuous function and the functional boundary conditions

$$
\begin{gathered}
L_{0}\left(u, u^{\prime}, u^{\prime \prime}, u(0)\right)=0 \\
L_{1}\left(u, u^{\prime}, u^{\prime \prime}, u^{\prime}(0)\right)=0 \\
L_{2}\left(u, u^{\prime}, u^{\prime \prime}, u^{\prime \prime}(0), u^{\prime \prime \prime}(0)\right)=0 \\
L_{3}\left(u, u^{\prime}, u^{\prime \prime}, u^{\prime \prime}(1), u^{\prime \prime \prime}(1)\right)=0
\end{gathered}
$$

where $L_{0}, L_{1}: C(I)^{3} \times \mathbb{R} \rightarrow \mathbb{R}$ and $L_{2}, L_{3}: C(I)^{3} \times \mathbb{R}^{2} \rightarrow \mathbb{R}$ are continuous functions, nondecreasing on all variables except the fourth one ( see [7]).

Lower and upper solutions for the above problem are defined as it follows:

Definition 2.1. A function $\alpha \in C^{4}(I)$ is a lower solution of problem (2.1)-(2.2) if:

$$
\alpha^{(i v)}(x) \geq f\left(x, \alpha(x), \alpha^{\prime}(x), \alpha^{\prime \prime}(x), \alpha^{\prime \prime \prime}(x)\right),
$$

and

$$
\begin{gathered}
L_{0}\left(\alpha, \alpha^{\prime}, \alpha^{\prime \prime}, \alpha(0)\right) \geq 0 \\
L_{1}\left(\alpha, \alpha^{\prime}, \alpha^{\prime \prime}, \alpha^{\prime}(0)\right) \geq 0 \\
L_{2}\left(\alpha, \alpha^{\prime}, \alpha^{\prime \prime}, \alpha^{\prime \prime}(0), \alpha^{\prime \prime \prime}(0)\right) \geq 0 \\
L_{3}\left(\alpha, \alpha^{\prime}, \alpha^{\prime \prime}, \alpha^{\prime \prime}(1), \alpha^{\prime \prime \prime}(1)\right) \geq 0
\end{gathered}
$$

The function $\beta \in C^{4}(I)$ is an upper solution of the problem (2.1)-(2.2) if the reversed inequalities hold.

Sufficient conditions for the existence of solution are given by the following theorem

Theorem 2.2. ([7], Thm 3.1) Suppose that there are upper and lower solutions of the problem (2.1)-(2.2), $\alpha(x)$ and $\beta(x)$, respectively, such that,

$$
\alpha(0) \leq \beta(0), \alpha^{\prime}(0) \leq \beta^{\prime}(0), \alpha^{\prime \prime}(x) \leq \beta^{\prime \prime}(x), \forall x \in[0,1]
$$


If $f:[0,1] \times \mathbb{R}^{4} \rightarrow \mathbb{R}$ is a continuous function satisfying Nagumo conditions in

$$
E_{*}=\left\{\begin{array}{c}
\left(x, y_{0}, y_{1}, y_{2}, y_{3}\right) \in[0,1] \times \mathbb{R}^{4}: \alpha(x) \leq y_{0} \leq \beta(x), \\
\alpha^{\prime}(x) \leq y_{1} \leq \beta^{\prime}(x), \alpha^{\prime \prime}(x) \leq y_{2} \leq \beta^{\prime \prime}(x)
\end{array}\right\}
$$

and

$$
f\left(x, \alpha, \alpha^{\prime}, y_{2}, y_{3}\right) \geq f\left(x, y_{0}, y_{1}, y_{2}, y_{3}\right) \geq f\left(x, \beta, \beta^{\prime}, y_{2}, y_{3}\right),
$$

for $\alpha(x) \leq y_{0} \leq \beta(x), \alpha^{\prime}(x) \leq y_{1} \leq \beta^{\prime}(x)$, in $[0,1]$, and fixed $\left(x, y_{2}, y_{3}\right) \in[0,1] \times \mathbb{R}^{2}$, then problem (2.1)-(2.2) has at least a solution $u(x) \in C^{4}([0,1])$, such that

$$
\alpha^{(i)}(x) \leq u^{(i)}(x) \leq \beta^{(i)}(x), \forall x \in[0,1], \text { for } i=0,1,2 .
$$

This result can be applied to the following multipoint problem

$$
\left\{\begin{array}{c}
u^{(i v)}(x)=-0.1(u(x))^{3}-0.1 \mid u^{\prime \prime 0.01 u^{\prime}(x)}+20 \sqrt[3]{\left|u^{\prime \prime \prime}(x)\right|} \\
\sum_{n=1}^{+\infty} a_{n}^{0} u\left(x_{n}\right)+\sum_{n=1}^{+\infty} b_{n}^{0} u^{\prime}\left(x_{n}\right)+\sum_{n=1}^{+\infty} c_{n}^{0} u^{\prime \prime}\left(x_{n}\right)-k u(0)=0 \\
\sum_{n=1}^{+\infty} a_{n}^{1} u\left(\widehat{x}_{n}\right)+\sum_{n=1}^{+\infty} b_{n}^{1} u^{\prime}\left(\widehat{x}_{n}\right)+\sum_{n=1}^{+\infty} c_{n}^{1} u^{\prime \prime}\left(\widehat{x}_{n}\right)-\eta u^{\prime}(0)=0 \\
u^{\prime \prime}(0)+2 u^{\prime \prime \prime}(0)=0 \\
u^{\prime \prime}(\mathbf{1})+u^{\prime \prime \prime}(1)=0
\end{array}\right.
$$

where $\sum_{n=1}^{+\infty} a_{n}^{i}, \sum_{n=1}^{+\infty} b_{n}^{i}, \sum_{n=1}^{+\infty} c_{n}^{i}$, for $i=0,1$, are positive convergent series to $a^{i}, b^{i}$ and $c^{i}$, respectively, $x_{n}, \widehat{x}_{n} \in[0,1], k \geq 7 a^{0}+8 b^{0}+8 c^{0}$ and $\eta \geq 3 a^{1}+3 b^{1}+\frac{8}{3} c^{1}$.

The functions $\alpha_{1}, \beta \in[0,1] \rightarrow \mathbb{R}$ given by

$$
\alpha_{1}(x)=-x^{2}-x-1 \text { and } \beta(x)=-x^{3}+4 x^{2}+3 x+1
$$

are, respectively, lower and upper solutions of (2.7) with

$$
\begin{aligned}
f\left(x, y_{0}, y_{1}, y_{2}, y_{3}\right) & =-0.1\left(y_{0}\right)^{3}-0.1\left|y_{2}-2\right| e^{0.01 y_{1}}+20 \sqrt[3]{\left|y_{3}\right|} \\
L_{0}\left(z_{1}, z_{2}, z_{3}, z_{4}\right) & =\sum_{n=1}^{+\infty} a_{n}^{0} z_{1}\left(x_{n}\right)+\sum_{n=1}^{+\infty} b_{n}^{0} z_{2}\left(x_{n}\right)+\sum_{n=1}^{+\infty} c_{n}^{0} z_{3}\left(x_{n}\right)-k z_{4} \\
L_{1}\left(z_{1}, z_{2}, z_{3}, z_{4}\right) & =\sum_{n=1}^{+\infty} a_{n}^{1} z_{1}\left(\widehat{x}_{n}\right)+\sum_{n=1}^{+\infty} b_{n}^{1} z_{2}\left(\widehat{x}_{n}\right)+\sum_{n=1}^{+\infty} c_{n}^{1} z_{3}\left(\widehat{x}_{n}\right)-\eta z_{4} \\
L_{2}\left(z_{1}, z_{2}, z_{3}, z_{4}, z_{5}\right) & =z_{4}+2 z_{5} \\
L_{3}\left(z_{1}, z_{2}, z_{3}, z_{4}, z_{5}\right) & =z_{4}+z_{5} .
\end{aligned}
$$

As

$$
\left|f\left(x, y_{0}, y_{1}, y_{2}, y_{3}\right)\right| \leq \varphi_{E_{*}}\left(y_{3}\right)=34.3+0.6 e^{0.08}+20 \sqrt[3]{\left|y_{3}\right|}
$$

then $f$ verifies Nagumo bilateral conditions in

$$
E_{*}=\left\{\left(x, y_{0}, y_{1}, y_{2}, y_{3}\right) \in[0,1] \times \mathbb{R}^{4}: \alpha_{1}^{(i)} \leq y_{i} \leq \beta^{(i)}, i=0,1,2\right\}
$$


and, by Theorem 2.2, there is a solution $u_{1}(x)$ of problem (2.7) such that, for every $x \in[0,1]$,

$$
\begin{aligned}
-x^{2}-x-1 & \leq u_{1}(x) \leq-x^{3}+4 x^{2}+3 x+1 \\
-2 x-1 & \leq u_{1}^{\prime}(x) \leq-3 x^{2}+8 x+3 \\
-2 & \leq u_{1}^{\prime \prime}(x) \leq-6 x+8, \quad \forall x \in[0,1] .
\end{aligned}
$$

Remark that the strips defined by (2.8) are relatively poor of information about $u_{1}$. However if the lower solution is chosen like $\alpha_{2}(x)=x^{2}$ then $f$ verifies the Nagumo conditions in

$$
E_{* *}=\left\{\left(x, y_{0}, y_{1}, y_{2}, y_{3}\right) \in[0,1] \times \mathbb{R}^{4}: \alpha_{2}^{(i)} \leq y_{i} \leq \beta^{(i)}, i=0,1,2\right\}
$$

and, by Theorem 2.2, there is a solution $u_{2}(x)$ of problem (2.7) such that, for every $x \in[0,1]$,

$$
\begin{aligned}
x^{2} & \leq u_{2}(x) \leq-x^{3}+4 x^{2}+3 x+1 \\
2 x & \leq u_{2}^{\prime}(x) \leq-3 x^{2}+8 x+3 \\
2 & \leq u_{2}^{\prime \prime}(x) \leq-6 x+8
\end{aligned}
$$

Indeed, this last case contains much more information about the solution: by (2.9), $u_{2}$ is non negative; by (2.10), $u_{2}$ is increasing; by (2.11), $u_{2}$ is convex and it can not be a straight line.

\subsection{Continuous model for the human spine}

In [1] it is considered the equation

$$
-\left(\phi\left(u^{\prime \prime \prime}(x)\right)\right)^{\prime}=f\left(x, u(x), u^{\prime}(x), u^{\prime \prime}(x), u^{\prime \prime \prime}(x)\right), \text { for a. e. } x \in J:=[a, b]
$$

with $\phi: \mathbb{R} \rightarrow \mathbb{R}$ an increasing and continuous function such that $\phi(0)=0, f: J \times \mathbb{R}^{4} \rightarrow \mathbb{R}$ a Carathéodory function and the functional boundary conditions

$$
\begin{aligned}
u(b) & =A, \quad u^{\prime}(b)=B, \quad A, B \in \mathbb{R}, \\
0 & =L_{1}\left(u, u^{\prime}, u^{\prime \prime}, u^{\prime \prime}(a), u^{\prime \prime \prime}(a)\right), \\
0 & =L_{2}\left(u, u^{\prime}, u^{\prime \prime}, u^{\prime \prime}(b), u^{\prime \prime \prime}(b)\right),
\end{aligned}
$$

where $L_{1}$ and $L_{2}$ are two continuous functions such that $L_{1}:(C(J))^{3} \times \mathbb{R}^{2} \rightarrow \mathbb{R}$ is nondecreasing on the first, third and fifth variables and nonincreasing on the second one, and $L_{2}:(C(J))^{3} \times \mathbb{R}^{2} \rightarrow \mathbb{R}$ is nondecreasing on the first and third variables and nonincreasing on the second and fifth ones.

Lower and upper solutions method allows to obtain the main result:

Theorem 2.3. ([1], Theorem 8) Assume that there is $\alpha$ a lower solution and $\beta$ an upper solution of problem (2.12)-(2.13) such that $\alpha^{\prime \prime}(x) \leq \beta^{\prime \prime}(x)$, for every $x \in J$. Suppose that 
$f: J \times \mathbb{R}^{4} \rightarrow \mathbb{R}$ is a continuous function satisfying a Nagumo-type condition in

$$
E_{*}=\left\{\begin{array}{c}
\left(x, y_{0}, y_{1}, y_{2}, y_{3}\right) \in J \times \mathbb{R}^{4}: \alpha^{(i)}(x) \leq y_{i} \leq \beta^{(i)}(x), i=0,2, \\
\beta^{\prime}(x) \leq y_{1} \leq \alpha^{\prime}(x)
\end{array}\right\}
$$

and, for $\left(x, y_{2}, y_{3}\right) \in J \times \mathbb{R}^{2}$ fixed, $\alpha(x) \leq y_{0} \leq \beta(x), \beta^{\prime}(x) \leq y_{1} \leq \alpha^{\prime}(x)$,

$$
f\left(x, \alpha(x), \alpha^{\prime}(x), y_{2}, y_{3}\right) \leq f\left(x, y_{0}, y_{1}, y_{2}, y_{3}\right) \leq f\left(x, \beta(x), \beta^{\prime}(x), y_{2}, y_{3}\right) .
$$

Then problem (2.12)-(2.13) has a solution u such that

$$
\alpha(x) \leq u(x) \leq \beta(x), \beta^{\prime}(x) \leq u^{\prime}(x) \leq \alpha^{\prime}(x), \alpha^{\prime \prime}(x) \leq u^{\prime \prime}(x) \leq \beta^{\prime \prime}(x), \forall x \in J .
$$

This theorem is applied to a continuous beam model of the human spine, to analyze its deformation under some axial and transverse loads. The total lateral displacement, $y(x)$, of the beam-column, with length $L$, is expressed as the sum of the initial lateral displacement, $y_{0}(x)$, known, and the lateral displacement due to the axial and transverse loads, $y_{1}(x)$, unknown, i.e.,

$$
y(x)=y_{0}(x)+y_{1}(x) .
$$

This displacement $y_{1}(x)$ is modeled by the differential equation

$$
E I y_{1}^{(4)}+P y_{1}^{\prime \prime}=q\left(y_{1}^{\prime \prime \prime}\right)-P y_{0}^{\prime \prime},
$$

where $E I$ is the flexural rigidity of the beam-column, $P$ the axial load and $q$ the transverse load. The boundary conditions for the unknown lateral displacement, $y_{1}(x)$, are given by

$$
y_{1}\left(\frac{L}{2}\right)=y_{1}^{\prime}\left(\frac{L}{2}\right)=y_{1}^{\prime \prime}\left(\frac{L}{2}\right)=0, y_{1}^{\prime \prime}\left(-\frac{L}{2}\right)=\max _{x \in J^{*}}\left\{y_{1}^{\prime \prime}(x)\right\} .
$$

meaning that the maximal value of the curvature will be attained at the left endpoint of the interval $J^{*}:=[-L / 2, L / 2]$.

As far as we know, it was the first time where such functional boundary conditions were considered in this type of models.

Under some technical assumptions, the functions

$$
\alpha(x)=\frac{a}{24} x^{4}-\frac{a}{24} L x^{3}-\frac{a}{20} L^{2} x^{2}+a L^{3} x-\frac{a}{2} L^{4}
$$

and

$$
\beta(x)=\frac{b}{24} x^{4}-\frac{b}{4} L x^{3}+\frac{b}{2} L^{2} x^{2}-2 b L^{3} x+b L^{4}
$$

are, respectively, lower and upper solutions of problem (2.15) - (2.16), for some $a, b>0$. As this problem is a particular case of (2.12)-(2.13), (see [1]), then, by Theorem 2.3, there is a solution of problem (2.15)-(2.16) such that the lateral displacement due to the axial and transverse loads verifies

$$
\alpha(x) \leq y_{1} \leq \beta(x), \beta^{\prime}(x) \leq y_{1}^{\prime} \leq \alpha^{\prime}(x), \alpha^{\prime \prime}(x) \leq y_{1}^{\prime \prime} \leq \beta^{\prime \prime}(x), \forall x \in J^{*} .
$$


More precisely, for $a=0.8, b=0.1, L=E I=P=1$, there is a solution $y_{1 *}(x)$ of problem (2.15)-(2.16) such that, for $x \in J^{*}$,

$$
\begin{aligned}
\frac{x^{4}}{30}-\frac{x^{3}}{30}-\frac{x^{2}}{25}+\frac{4 x}{5}-\frac{2}{5} & \leq y_{1 *}(x) \leq \frac{x^{4}}{240}-\frac{x^{3}}{40}+\frac{x^{2}}{20}-\frac{x}{5}+\frac{1}{10}, \\
\frac{x^{3}}{60}-\frac{3 x^{2}}{40}+\frac{x}{10}-\frac{1}{5} & \leq y_{1 *}^{\prime}(x) \leq \frac{2 x^{3}}{15}-\frac{x^{2}}{10}-\frac{2 x}{25}+\frac{4}{5} \\
\frac{2 x^{2}}{5}-\frac{x}{5}-\frac{2}{25} & \leq y_{1 *}^{\prime \prime}(x) \leq \frac{x^{2}}{20}-\frac{3 x}{20}+\frac{1}{10} .
\end{aligned}
$$

Remark that, from (2.17), it is clear that the displacement of the beam, $y_{1 *}(x)$, under the referred forces, is not a linear function.

\section{THIRD ORDER PERIODIC PROBLEM}

The branches used to locate the solution and/or its derivatives can be defined with ordered ("well" ordered or in "reversed" order) or non ordered lower and upper solutions. This last case can provide additional information about the solution and generalizes the admissible candidates to lower and upper functions, as it can be seen in the study of the third order nonlinear periodic problem, composed by the fully differential equation

$$
u^{\prime \prime \prime}(x)=f\left(x, u(x), u^{\prime}(x), u^{\prime \prime}(x)\right)
$$

where $x \in[0,1], f:[0,1] \times \mathbb{R}^{3} \rightarrow \mathbb{R}$ is a continuous function, and the periodic boundary conditions

$$
u(0)-u(1)=u^{\prime}(0)-u^{\prime}(1)=u^{\prime \prime}(0)-u^{\prime \prime}(1)=0 .
$$

The existence and location result for this problem will assume an unilateral Nagumotype condition, to obtain an a priori bound on the second derivative of all possible solutions of (3.1):

Given a subset $E \subset[0,1] \times \mathbb{R}^{3}$, a function $f:[0,1] \times \mathbb{R}^{3} \rightarrow \mathbb{R}$ is said to satisfy a Nagumo-type condition in $E$ if there exists $\varphi \in C\left(\mathbb{R}_{0}^{+},[k,+\infty[)\right.$, with $k>0$, such that

$$
f(x, y, z, w) \leq \varphi(|w|)
$$

or

$$
f(x, y, z, w) \geq-\varphi(|w|)
$$

for all $x \in[0,1]$ and all $(x, y, z, w) \in E$, and

$$
\int_{0}^{+\infty} \frac{s}{\varphi(s)} d s=+\infty .
$$

Lemma 3.1. ([5], Lemma 2) Let $f:[0,1] \times \mathbb{R}^{3} \longmapsto \mathbb{R}$, be a continuous function satisfying Nagumo conditions (3.3) and (3.5) in

$$
E=\left\{(x, y, z, w) \in[0,1] \times \mathbb{R}^{3}: \Gamma_{1}(x) \leq y \leq \Gamma_{2}(x), \gamma_{1}(x) \leq z \leq \gamma_{2}(x)\right\}
$$


with $\Gamma_{1}, \Gamma_{2}, \gamma_{1}, \gamma_{2} \in C([0,1], \mathbb{R})$ satisfying $\Gamma_{1}(x) \leq \Gamma_{2}(x)$ and $\gamma_{1}(x) \leq \gamma_{2}(x)$, for every $x \in[0,1]$. Then, for every $\rho>0$ there exists $r>0$ (depending on $\gamma_{1}, \gamma_{2}, \varphi, \rho$ ) such that for every solution $u(x)$ of $(3.1)$, with

$$
u^{\prime \prime}(0) \leq \rho, \quad u^{\prime \prime}(1) \geq-\rho
$$

and

$$
\Gamma_{1}(x) \leq u^{\prime}(x) \leq \Gamma_{2}(x), \gamma_{1}(x) \leq u^{\prime}(x) \leq \gamma_{2}(x), \forall x \in[0,1]
$$

verifies $\left\|u^{\prime \prime}\right\| \leq r$.

Remark 3.2. The previous Lemma still holds if condition (3.4) is assumed and (3.6) is replaced by

$$
u^{\prime \prime}(0) \geq-\rho, u^{\prime \prime}(1) \leq \rho .
$$

Non ordered upper and lower solutions are defined as a pair of functions:

Definition 3.3. The functions $\alpha, \beta \in C^{3}([0,1])$ are a pair of lower and upper solutions, respectively, of problem (3.1)-(3.2) if:

(i) $\alpha^{\prime \prime \prime}(x) \geq f\left(x, \bar{\alpha}(x), \alpha^{\prime}(x), \alpha^{\prime \prime}(x)\right)$

with

$$
\bar{\alpha}(x)=\min _{x \in[0,1]}\{\alpha(x), \beta(x)\}
$$

(ii) $\bar{\alpha}(0)=\bar{\alpha}(1), \alpha^{\prime}(0)=\alpha^{\prime}(1), \alpha^{\prime \prime}(0) \geq \alpha^{\prime \prime}(1)$;

(iii) $\beta^{\prime \prime \prime}(x) \leq f\left(x, \bar{\beta}(x), \beta^{\prime}(x), \beta^{\prime \prime}(x)\right)$

with

$$
\bar{\beta}(x)=\max _{x \in[0,1]}\{\alpha(x), \beta(x)\}
$$

(iv)

$$
\bar{\beta}(0)=\bar{\beta}(1), \beta^{\prime}(0)=\beta^{\prime}(1), \beta^{\prime \prime}(0) \leq \beta^{\prime \prime}(1) .
$$

The existence and location result for problem (3.1)-(3.2) is given by next theorem:

Theorem 3.4. Assume that $\alpha, \beta \in C^{3}([0,1])$ are a pair of lower and upper solutions of (3.1)-(3.2), respectively, such that

$$
\alpha(0) \geq \beta(0), \alpha(1) \leq \beta(1), \alpha^{\prime}(x) \leq \beta^{\prime}(x), \forall x \in[0,1] .
$$

Let $f:[0,1] \times \mathbb{R}^{3} \rightarrow \mathbb{R}$ be a continuous function verifying unilateral Nagumo-type conditions ( 3.3), or (3.4), and (3.5), in

$$
E_{*}=\left\{(x, y, z, w) \in[0,1] \times \mathbb{R}^{3}: \bar{\alpha}(x) \leq y \leq \bar{\beta}(x), \alpha^{\prime}(x) \leq z \leq \beta^{\prime}(x)\right\}
$$

and

$$
f(x, \bar{\alpha}(x), z, w) \geq f(x, y, z, w) \geq f(x, \bar{\beta}(x), z, w),
$$

for $(x, z, w) \in[0,1] \times \mathbb{R}^{2}$ and $\bar{\alpha}(x) \leq y \leq \bar{\beta}(x)$.

Then problem (3.1)-(3.2) has at least a periodic solution $u \in C^{3}([0,1])$ such that

$$
\bar{\alpha}(x) \leq u(x) \leq \bar{\beta}(x) \text { and } \alpha^{\prime}(x) \leq u^{\prime}(x) \leq \beta^{\prime}(x), \forall x \in[0,1]
$$


Proof. For $\lambda \in[0,1]$, consider the auxiliary modified problem

$$
u^{\prime \prime \prime}(x)=\lambda f\left(x, \bar{\delta}(x, u(x)), \delta\left(x, u^{\prime}(x)\right), u^{\prime \prime}(x)\right)+u^{\prime}(x)-\lambda \delta\left(x, u^{\prime}(x),\right.
$$

where the continuous functions $\bar{\delta}, \delta: \mathbb{R}^{2} \rightarrow \mathbb{R}$ are given by

$$
\bar{\delta}(x, y)=\left\{\begin{array}{cc}
\bar{\beta}, & y>\bar{\beta} \\
y, & \bar{\alpha} \leq y \leq \bar{\beta} \\
\bar{\alpha}, & y<\bar{\alpha}
\end{array} \quad \delta(x, z)=\left\{\begin{array}{cc}
\beta^{\prime}, & z>\beta^{\prime} \\
z, & \alpha^{\prime} \leq z \leq \beta^{\prime} \\
\alpha^{\prime}, & z<\alpha^{\prime}
\end{array}\right.\right.
$$

$\bar{\alpha}$ and $\bar{\beta}$ are defined in Definition 3.3, with the boundary conditions

$$
u(0)=\lambda u(1), u^{\prime}(0)=u^{\prime}(1), u^{\prime \prime}(0)=u^{\prime \prime}(1) .
$$

Take $r_{1}>0$ such that, for every $x \in[0,1]$,

$$
\begin{gathered}
-r_{1}<\alpha^{\prime}(x) \leq \beta^{\prime}(x)<r_{1}, \\
f\left(x, \bar{\alpha}(x), \alpha^{\prime}(x), 0\right)-r_{1}-\alpha^{\prime}(x)<0, \\
f\left(x, \bar{\beta}(x), \beta^{\prime}(x), 0\right)+r_{1}-\beta^{\prime}(x)>0,
\end{gathered}
$$

and, for every solution $u$ of (3.9)-(3.10),

$$
\left|u^{\prime \prime}(1)\right|<r_{1} .
$$

The arguments follow standard techniques in lower and upper solutions method and they are omitted. For reader's convenience it is only sketched the main steps (for further details see, for instance, $[5,6])$ :

- Every solution $u$ of the problem (3.9)-(3.10) satisfies, in [0,1],

$$
\left|u^{\prime}(x)\right|<r_{1} \text { and }|u(x)|<r_{0},
$$

with $r_{1}$ given above and $r_{0}=|u(0)|+r_{1}$, independently of $\lambda \in[0,1]$.

- There exists $R>0$ such that every solution $u$ of the problem (3.9)-(3.10) satisfies

$$
\left|u^{\prime \prime}(x)\right|<R, \forall x \in[0,1]
$$

independently of $\lambda \in[0,1]$.

Remark that if $f$ verifies (3.3) then the function $F_{\lambda}:[0,1] \times \mathbb{R}^{3} \rightarrow \mathbb{R}$ defined by

$$
F_{\lambda}(x, y, z, w)=\lambda f(x, \bar{\delta}(x, y), \delta(x, z), w)+z-\delta(x, z)
$$

satisfies

$$
F_{\lambda}(x, y, z, w) \leq \lambda \varphi(|w|)+r_{1}+\mid \delta(x, z) \leq \varphi(|w|)+2 r_{1} .
$$

Therefore $F_{\lambda}$ verifies (3.3) with $\varphi(w)$ replaced by $\bar{\varphi}(w):=\varphi(w)+2 r_{1}$, independently of $\lambda \in[0,1]$, and, by (3.10) and (3.11), condition (3.6) holds.

Analogously if $f$ verifies (3.4). 
- For $\lambda=1$ problem (3.9)-(3.10) has a solution $u_{1}(x)$.

- $u_{1}(x)$ is a solution of (3.1)-(3.2).

This type of non ordered lower and upper solutions allows to evaluate at least a value of the solution at an interior point.

Corollary 3.5. If assumptions of Theorem 3.4 hold and

$$
\alpha(0)>\beta(0), \alpha(1)<\beta(1)
$$

then there is $c \in] 0,1[$ such that

$$
\alpha(c)=u(c)=\beta(c)
$$

Proof. From Theorem 3.4 there is a solution $u(x)$ such that

$$
\bar{\alpha}(x) \leq u(x) \leq \bar{\beta}(x), \forall x \in[0,1]
$$

Therefore it will be enough to prove that there is $c \in] 0,1[$ such that $\bar{\alpha}(c)=\bar{\beta}(c)$.

As the function $(\alpha-\beta)(x)$ is continuous and

$$
\alpha(0)-\beta(0)>0>\alpha(1)-\beta(1)
$$

then there is $c \in] 0,1[$ such that $\alpha(c)=\beta(c)$, that is, $\bar{\alpha}(c)=\bar{\beta}(c)$.

Example: Consider the third order differential equation

$$
u^{\prime \prime \prime}(x)=-u(x)^{3}+4 u^{\prime}(x)^{5}-\frac{1}{2} u^{\prime \prime}(x)^{2}
$$

with the periodic boundary conditions (3.2).

The continuous functions $\alpha, \beta: \mathbb{R} \rightarrow \mathbb{R}$ given by

$$
\alpha(x)=-x+1, \beta(x)=x
$$

are a pair of lower and upper solutions, respectively, of problem (3.13)-(3.2) verifying (3.7).

The function

$$
f(x, y, z, w)=-y^{3}+4 z^{5}-\frac{1}{2} w^{2}
$$

is continuous, verifies Nagumo-type conditions (3.3) and (3.5), with $\varphi(w)=5+\frac{w^{2}}{2}$ in

$$
E=\left\{(x, y, z, w) \in[0,1] \times \mathbb{R}^{3}: \bar{\alpha}(x)<y<\bar{\beta}(x),-1<z<1\right\}
$$

and satisfies (3.8). 
By Theorem 3.4 there is a solution $u(x)$ of problem (3.13)-(3.2), such that

$$
x \leq u(x) \leq-x+1 \quad \text { if } \quad 0 \leq x \leq \frac{1}{2}
$$

and

$$
-x+1 \leq u(x) \leq x \quad \text { if } \quad \frac{1}{2} \leq x \leq 1 .
$$

Remark that, by (3.13) and the location zone, this solution $u$ is non trivial and $u\left(\frac{1}{2}\right)=\frac{1}{2}$. Moreover, from this location part it may be said that problem (3.13)-(3.2) has infinite periodic solutions. In fact, for each $k \in \mathbb{Z}$, the functions

$$
\alpha_{k}(x)=-x+k+1, \beta_{k}(x)=x+k
$$

are a pair of lower and upper solutions, respectively, of problem (3.13)-(3.2) verifying (3.7). Therefore, by Theorem 3.4 , for each $k \in \mathbb{Z}$ there a solution $u_{k}$ verifying

$$
\begin{array}{lll}
x+k \leq u_{k}(x) \leq-x+k+1 & \text { if } & 0 \leq x \leq \frac{1}{2}, \\
-x+k+1 \leq u_{k}(x) \leq x+k & \text { if } & \frac{1}{2} \leq x \leq 1
\end{array}
$$

and $u_{k}\left(\frac{1}{2}\right)=\frac{1}{2}+k$.

\section{REFERENCES}

1. A. Cabada and F. Minhós, Fully nonlinear fourth-order equations with functional boundary conditions, J. Math. Anal. Appl. 340 (2008) 239-251.

2. A. Cabada, R. Pouso and F. Minhós, Extremal solutions to fourth-order functional boundary value problems including multipoint condition, Nonlinear Analysis: Real World Appl. doi:10.1016/j.nonrwa.2008.03.026

3. S. Chen, W. Ni and C. Wang, Positive solutions of fourth order ordinary differential equation with four-point boundary conditions. Appl. Math. Letters 19 (2006) 161-168.

4. D. Franco, D. O'Regan and J. Perán, Fourth-order problems with nonlinear boundary conditions, J. Comput. Appl. Math. 174 (2005) 315-327.

5. M. R. Grossinho, F. Minhós and A. I. Santos, Solvability of some third-order boundary value problems with asymmetric unbounded nonlinearities, Nonlinear Anal. 62 (2005) 1235-1250.

6. F. Minhós Periodic third order problems: existence and location results, Proc. of the 6th International Conference On Differential Equations and Dynamical Systems, (2009) 122-126, DCDIS A Supplement, Watam Press.

7. F. Minhós and J. Fialho On the solvability of some fourth-order equations with functional boundary conditions (To appear in Proc. of Seventh International Conference on Dynamical Systems, Differential Equations and Applications, 2008, AIMS).

8. S. Mukhigulashvili, On a periodic boundary value problem for third order linear functional differential equations. Nonlinear Anal., 66 (2007) 527-535.

9. J. Nieto, Periodic solutions for third order ordinary differential equations, Comment. Math. Univ. Carolinae., 32/3 (1991) 495-499

10. I. Rachunková, Periodic boundary value problems for third order differential equations, Math. Slovaca, 41/3 (1991) 241-248.

11. Q. Zhang, S. Chen and J. Lü, Upper and lower solution method for fourth-order four-point boundary value problems, J. Comp. Appl. Math. 196 (2006) 387-393. 